\title{
Contribuições para a evolução do gerenciamento de resíduos sólidos urbanos no Brasil com base na experiência Européia
}

\author{
Contribution to the development of municipal solid \\ waste management in Brazil based on European experience
}

Camille Ferreira Mannarino', João Alberto Ferreira², Mauro Gandolla ${ }^{3}$

\begin{abstract}
RESUMO
Esse trabalho tem como objetivo apresentar dados relevantes sobre 0 gerenciamento de resíduos sólidos urbanos (RSU) na Europa de forma a se estabelecer paralelos e subsidiar a discussão no Brasil. É apresentada uma contextualização da situação atual da gestão dos resíduos sólidos urbanos no Brasil, onde os efeitos da Política Nacional de Resíduos Sólidos já se fazem sentir, sobretudo com o aumento da disposição final de RSU em aterros sanitários, predominantemente nas regiões metropolitanas. A reciclagem no país, em grande parte, ainda baseia-se na coleta informal por catadores ou na ação de organizações privadas. Na Europa, a partir da Diretiva 1999/31/EC, que estabeleceu metas para a disposição de resíduos em aterros sanitários, e da Diretiva 94/62/EC, sobre recolhimento deembalagens, a gestão de RSU sealterou profundamente. São apresentados dados das tecnologias predominantes de tratamento e disposição final de RSU e alguns mecanismos para implementação da mudança de cenário na Europa. O exemplo suíço é detalhado, mostrando como a cobrança de tarifas impulsiona as ações da população. É preciso reconhecer que o gerenciamento de resíduos sólidos urbanos RSU tem que ser planejado da coleta até o destino final, utilizando e integrando diferentes tecnologias de tratamento, de acordo com as características locais. É relevante para o Brasil a avaliação criteriosa das soluções disponíveis, do ponto de vista técnico e operacional, considerando fortemente a sua aplicabilidade local.
\end{abstract}

Palavras-chave: gerenciamento integrado de resíduos; tratamento de resíduos; disposição final de resíduos; incineração; Europa; Suiça.

\begin{abstract}
This paper aims to present relevant data on municipal solid waste (MSW) management in Europe in order to establish parallel and support the discussion in Brazil. The current situation of municipal solid waste management in Brazil is presented. It is possible to see some consequences of the Brazilian National Solid Waste Policy, especially concerning the increase of MSW disposal in landfills, mostly in metropolitan areas. Recycling in the country is largely based on informal collection by waste pickers or action by private organizations. In Europe the MSW management has largely changed as consequence of the Directive 1999/31/EC, which set targets for waste disposal in landfills, and the Directive 94/62/EC, about packaging collection. This paper presents data about predominant MSW treatment and disposal technologies and some mechanisms applied to implementing the scenario changes in Europe. The Swiss example is detailed showing how the charging of fees drives people's actions. It must be recognized that the MSW management has to be planned from collection to final destination, using and integrating different treatment technologies, in accordance with local characteristics. It is relevant to Brazil a carefully evaluation, both technically and operationally, of the available solutions considering their local applicability.
\end{abstract}

Keywords: integrated solid waste management; waste treatment; waste disposal; incineration; Europe; Switzerland.

'Doutora em Saúde Pública e Meio Ambiente pela Escola Nacional de Saúde Pública Sergio Arouca da Fundação Oswaldo Cruz (ENSP/FIOCRUZ). Pesquisadora em saúde pública no Departamento de Saneamento e Saúde Ambiental/ENSP/FIOCRUZ - Rio de Janeiro (RJ), Brasil.

${ }^{2}$ Doutor em Saúde Pública pela ENSP/FIOCRUZ Oswaldo Cruz. Pesquisador visitante no Departamento de Engenharia Sanitária e do Meio Ambiente/UERJ - Rio de Janeiro (RJ), Brasil. ${ }^{3}$ Engenheiro Mecânico pelo Swiss Federal Institute of Technology in Zürich (Suíça). Diretor da empresa ECONS S.A. (Suíça) - Bioggio (TI), Suíça.

Endereço para correspondência: Camille Ferreira Mannarino - Rua Paissandu, 334/4O2 - Laranjeiras - 22210-080 - Rio de Janeiro (RJ), Brasil - E-mail: camille.mannarino@ensp.fiocruz.br Recebido: 26/02/15 - Aceito: 18/09/15 - Reg. Abes: 146475 


\section{INTRODUÇÃO}

O cenário de gestão de resíduos sólidos urbanos no Brasil está passando por mudanças nos últimos anos, sobretudo após a aprovação da Política Nacional de Resíduos Sólidos, instituída pela Lei Federal n 12.305, de 02 de agosto de 2010 (BRASIL, 2010). Nela, estão estabelecidas as diretrizes relativas à gestão integrada e ao gerenciamento de resíduos sólidos, as responsabilidades dos geradores e do poder público e os instrumentos econômicos aplicáveis, tendo assimilado conceitos vigentes em países desenvolvidos, particularmente na Europa.

Como decorrência da Política Nacional de Resíduos Sólidos, têm sido estruturadas ações no nível federal de apoio e incentivo à gestão adequada dos resíduos sólidos no país, com destaque para a destinação final em aterros sanitários e as tratativas para elaboração dos acordos setoriais para a logística reversa. Percebe-se ainda uma participação maior dos estados na transferência de recursos para os municípios e na busca de estruturação de soluções consorciadas. Algumas mudanças já podem ser percebidas em dados consolidados por pesquisas realizadas na área.

A quantidade de resíduos sólidos urbanos coletados no Brasil foi estimada em aproximadamente 189 mil de toneladas por dia, para o ano de 2013 (ABRELPE, 2014). A geração de resíduos sólidos urbanos no país vem aumentando a cada ano, reflexo, sobretudo, do crescimento da economia, que permite à população o acesso a novos bens e produtos, e da grande presença de materiais descartáveis entre os produtos consumidos. Entre os anos de 2012 e 2013, o crescimento registrado na geração estimada de resíduos sólidos urbanos foi de $4,1 \%$, índice superior à taxa de crescimento populacional urbano do país, 3,7\% no mesmo período (ABRELPE, 2014).

No ano de 2013,42\%, em peso, dos resíduos sólidos urbanos coletados no Brasil eram dispostos em locais inadequados, em lixões e aterros controlados, causando impactos ambientais no solo, nas águas, no ar e na saúde pública (ABRELPE, 2014). Se, no ano de 2013,58\% dos resíduos sólidos urbanos coletados foram destinados corretamente, esse número correspondia a 36\% no ano 2000 (ABRELPE, 2003; 2014). Destaca-se que as melhorias na destinação final dos RSU estão sobretudo concentradas nas regiões metropolitanas e grandes cidades do país.

De acordo com dados das Pesquisas Nacionais de Saneamento Básico realizadas pelo IBGE, do total de unidades de destinação final de resíduos sólidos no Brasil, apenas 1,1\% eram aterros sanitários em 1989. No ano 2000, 17,3\% dessas unidades eram aterros sanitários e, em 2008, 27,7\% (IBGE, 2010).

A realidade das regiões e municípios brasileiros é bastante diferente em relação à capacidade de investimento na gestão de resíduos sólidos. Entretanto, as exigências definidas pela Política Nacional de Resíduos Sólidos são as mesmas para todo o país. No tocante ao destino final de resíduos sólidos, a legislação federal definiu que "a disposição final ambientalmente adequada dos rejeitos (...) deverá ser implantada em até 4 (quatro) anos após a data de publicação desta Lei", prazo que se encerraria em agosto de 2014 (BRASIL, 2010). Dado que grande parte dos municípios brasileiros ainda mantinha lixões como destino final para seus resíduos nessa data, uma alteração na lei está sendo discutida, com aumento do prazo para o cumprimento dessa meta.

Outra exigência definida pela Política Nacional de Resíduos Sólidos refere-se à implantação de sistemas de coleta seletiva, visando à recuperação e à reciclagem de materiais (BRASIL, 2010). Pesquisa realizada no ano de 2013 indica que 62\% dos municípios brasileiros possuem iniciativas de coleta seletiva, sendo essas predominantemente encontradas em municípios com mais de 100 mil habitantes (ABRELPE, 2014). O estudo ressalta, entretanto, que tais iniciativas, muitas vezes, resumem-se à existência de pontos de entrega voluntária de resíduos ou convênio com cooperativas de catadores e que, em muitos municípios, as atividades de coleta seletiva não abrangem a totalidade de sua área urbana.

Dificuldades para a efetiva implantação de um sistema de reciclagem de materiais no Brasil estão relacionadas também a: falta de adesão da população à coleta seletiva; pouca participação do setor industrial no desenvolvimento de um sistema de logística reversa; inexistência de locais adequados para separação dos resíduos por tipo de material; além de, em muitos casos, longas distâncias entre os centros geradores de resíduos e as indústrias de processamento e reciclagem de materiais concentradas, principalmente, nas regiões Sul e Sudeste do país.

Embora muitos municípios ainda precisem resolver problemas básicos na gestão de resíduos sólidos, como aumento na abrangência e eficiência da coleta, adequação do local de disposição final de resíduos e/ou implantação de sistemas de coleta seletiva, existem aqueles, sobretudo cidades de grande porte e com maior disponibilidade de recursos financeiros e tecnológicos, que já se encontram em situação mais bem estruturada nessa área e que começam a buscar tecnologias mais avançadas de tratamento de resíduos.

Por esta razão, considera-se importante um melhor conhecimento da gestão de resíduos sólidos urbanos em países desenvolvidos, das tecnologias utilizadas e experiências negativas, para que se amplie a reflexão da gestão no país tendo em vista as enormes desigualdades regionais e as dificuldades para implementação de um modelo que se adeque a tais desigualdades.

O presente trabalho tem como objetivo apresentar dados relevantes relativos ao gerenciamento de resíduos sólidos urbanos na Europa, sobretudo na Suíça, de forma a contribuir para a avaliação, no Brasil, das tecnologias e modelos lá adotados, visando a evolução do setor de resíduos no país.

\section{METODOLOGIA}

O trabalho foi desenvolvido buscando-se relacionar as características principais da gestão de resíduos sólidos urbanos no Brasil e na Europa e mostrar aspectos da gestão nesse continente que contextualizam a sua evolução na área. 
Nesse sentido, são apresentados e discutidos dados secundários de diversas pesquisas e fontes que consolidam informações sobre gestão de resíduos na Europa, buscando-se traçar um paralelo com a situação brasileira.

As discussões foram conformadas a partir da experiência dos autores, enquanto consultores para gestão de resíduos no Brasil e na Europa.

\section{RESULTADOS E DISCUSSÃO}

\section{A situação da gestão de resíduos sólidos urbanos na Europa}

De acordo com dados do Statistical Office of the European UnionEurostat, no ano de 2012, foram produzidas aproximadamente 248 milhões de toneladas de resíduos sólidos urbanos nos 28 estadosmembros da União Européia, aproximadamente 680 mil toneladas diárias (EUROSTAT, 2014a).

Em 23 dos estados da União Européia, a quantidade de resíduos sólidos urbanos gerados per capita aumentou entre os anos de 1995 e 2009 (EUROSTAT, 2011). Crescimentos anuais expressivos na geração de resíduos, de mais de 3,0\%, foram observados em Malta, Grécia e Dinamarca nesse período (EUROSTAT,2011).Entretanto,observa-se que, a partir do ano de 2008, a geração per capita média vem se reduzindo entre estados da União Européia. Em 2008, a média anual de resíduos gerados por pessoa era de $520 \mathrm{~kg}\left(1,42 \mathrm{~kg} \cdot \mathrm{hab}^{-1} \cdot \mathrm{dia}^{-1}\right)$ enquanto que, em 2012, esse valor passou a $492 \mathrm{~kg}\left(1,35 \mathrm{~kg} \cdot \mathrm{hab}^{-1} \cdot \mathrm{dia}^{-1}\right)$ (EUROSTAT, 2014a).

Essa redução pode estar relacionada à crise econômica vigente em alguns países da Europa, que implica em redução de salários e do consumo, resultando em um decaimento da produção de resíduos. Uma maior consciência da população sobre o tema, devido à políticas públicas e leis lá adotadas também contribui para esse fato.

Como formas de tratamento e destino final dos resíduos sólidos urbanos na União Européia, no ano de 2012, registrou-se que $27 \%$ dos resíduos eram encaminhados para reciclagem, $15 \%$ para compostagem, $24 \%$ para incineração e 34\% destinados para aterros sanitários (EUROSTAT, 2014b).

Entre os países integrantes da União Europeia, com a evolução da gestão de resíduos sólidos, percebe-se uma tendência de redução de destinação de resíduos sólidos urbanos para aterros sanitários, que, entre os anos de 1995 e 2012, foi de 42\% em peso (EUROSTAT, 2014a). A quantidade de resíduos encaminhados para incineração, nesse período, teve comportamento oposto, apresentando aumento de $80 \%$ (EUROSTAT, 2014a). A reciclagem e os tratamentos biológicos (compostagem e digestão anaeróbia) tiveram crescimento bastante expressivo, respectivamente 162 e
149\% em relação ao peso de resíduos encaminhados para cada um (EUROSTAT, 2014a).

As mudanças na gestão de resíduos sólidos urbanos na Europa podem ser atribuidas em grande parte à legislação local, que determina metas para a evolução do setor. Como destaque na legislação européia, deve ser citada a Diretiva 94/62/EC, que determinou que 50\% das embalagens (e seus resíduos) colocadas no mercado deveriam ser recolhidas até o ano de 2001 (UE, 1994). Essa meta foi revisada, prevendo que $60 \%$ das embalagens (e seus resíduos) deveriam ser recolhidas até o final de 2008.

Destaca-se também a Diretiva 1999/31/EC, que trata da disposição de resíduos em aterros (UE, 1999). Essa lei determina metas progressivas para a redução da disposição de matéria orgânica biodegradável diretamente nos aterros sanitários, que deve ser no máximo 35\% da quantidade total (peso) dos resíduos biodegradáveis produzidos em 1995, até julho de 2016 (UE, 1999).

Com a necessidade de recolhimento de embalagens e a limitação da possibilidade de disposição final de matéria orgânica nos aterros, houve a necessidade de desenvolvimento de estratégias e investimentos em reciclagem de materiais e tratamento da fração orgânica. Como consequência, o aumento dos percentuais de tratamento de resíduos e a redução da disposição em aterros.

Além da legislação, instrumentos econômicos também se mostram importantes na mudança de gestão de resíduos na Europa. Adotado em alguns países, o princípio da causalidade atribui responsabilidades ao gerador pelos resíduos gerados. Uma importante expressão desse princípio é a taxa cobrada por saco de lixo gerado.

Resíduos recicláveis, como plástico politereftalato de etileno (PET), vidros, papeis e metais, além de vegetais crus, que podem ser tratados por compostagem ou digestão anaeróbia, são recolhidos sem cobrança de taxa adicional. Os materiais recicláves podem ser dispostos em containeres localizados em espaços públicos ou entregues em estabelecimentos comerciais. Os demais resíduos a serem disponibilizados para coleta devem ser acondicionados em sacos plásticos específicos, pelos quais se paga uma taxa.

Na Suíça, cada saco de 35 L custa entre 2 e 3 Francos Suíços (SFr), ou aproximadamente entre 6,08 e 9,11 Reais (R\$) (taxa de conversão utilizada: 1 SFr = R\$3,04, em 23 de fevereiro de 2015). O valor elevado de cada saco de lixo incentiva à população a separar os resíduos recicláveis e tratáveis biologicamente, a fim de reduzir os gastos para o recolhimento dos resíduos gerados.

Outro instrumento econômico importante é a taxa de eliminação antecipada ou contribuição antecipada para reciclagem, embutida no preço que o consumidor paga por alguns produtos. Essa taxa é recolhida pelos comerciantes para auxiliar no financiamento do sistema de coleta seletiva. 
Como exemplos dessa taxa, paga-se, na Suíça, 1,8 centavos de Francos Suíços, cerca de 5,5 centavos de Real, em cada garrafa plástica; 0,85 centavos de Francos Suíços (2,6 centavos de Real) em cada lata de até 1,5 L; SFr 1,2 ( $\mathrm{R} \$ 3,65)$ por lâmpada fluorescente; 0,60 centavos de Francos Suíços $(\mathrm{R} \$ 1,82)$ por garrafa de vidro; SFr 8,0 ( $\mathrm{R} \$ 24,32)$ em cada impressora e 5,0 centavos de Francos Suíços (15 centavos de Real) em cada pilha AA (OFEV, 2013).

De acordo com o Instituto Federal do Meio Ambiente da Suíça OFEV, no ano de 2012, a receita da taxa de eliminação antecipada para pilhas foi de SFr 14 milhões ( $\mathrm{R} \$ 42.560 .000,00)$, o que contribuiu para o recolhimento e reciclagem de 2.500 toneladas de pilhas (OFEV,2013).

É preciso ainda considerar que a redução da destinação final de resíduos para aterros sanitários está também ligada aos custos para execução dos dispositivos de controle ambiental e do monitoramento dos mesmos exigidos pela legislação local.

A Diretiva 1999/31/EC determina que o monitoramento deve ser realizado durante toda a operação de um aterro e por um período de pelo menos 30 anos após o encerramento do mesmo ou enquanto as autoridades competentes considerarem que o aterro pode apresentar perigo para o ambiente, sendo os seus custos de responsabilidade do operador do aterro (UE, 1999).

De acordo com a legislação europeia, o monitoramento dessas áreas deve abranger ao menos os seguintes itens: dados meteorológicos; volume e composição do lixiviado; volume e composição das águas superficiais; emissões de gases e pressão atmosférica; níveis e composição das águas subterrâneas, estrutura e composição do aterro; recalques do aterro (UE, 1999).

Nos aterros sanitários, existe a necessidade de tratamento do lixiviado e do biogás produzidos (variáveis de acordo com o clima), além do controle de acidentes, durante a sua operação, encerramento e pós-encerramento. Esses custos usualmente são maiores do que os de monitoramento e precisam estar previstos no planejamento de um aterro sanitário.

Destaca-se que, na Suíça, existe uma obrigação legal de incineração de todos os resíduos que não possam passar por processos de valorização por reciclagem, incluindo tratamentos biológicos da matéria orgânica (SUISSE, 1990). Nesse país, desde 2004, não são encaminhados resíduos sólidos urbanos para aterros sanitários, que recebem apenas os rejeitos dos tratamentos desses resíduos (OFEV, 2011).

\section{A gestão de resíduos sólidos como parte do planejamento urbano}

Diversas legislações européias têm como princípio a minimização dos impactos negativos da produção e gestão dos resíduos sólidos na saúde humana e no ambiente, sendo estabelecidas regras para tornar a valorização e a eliminação dos resíduos mais sustentáveis (UE, 1994; 1999; 2000; 2006; 2008).

Uma visão moderna de gestão de resíduos vigente na Europa sugere que esse tema deve ser tratado como parte do planejamento urbano de uma localidade e não como uma questão à parte (GANDOLLA \& QUITIAN, 2005; GANDOLLA et al., 2009).

Todas as etapas de um sistema de gestão de resíduos, desde o acondicionamento, passando pela acumulação, coleta, transporte, valorização, tratamento e destino final, devem ser integradas não somente entre si, mas também com a estrutura urbana onde elas estão inseridas (GANDOLLA \& QUITIAN, 2005; GANDOLLA et al., 2009).

Tomando-se como exemplo o local de destinação final dos resíduos, ele não deve ser consideradoapenas um local de confinamento dos resíduos longe da população. É importante que esse local seja visto como um local onde os resíduos serão reinseridos no seu ciclo natural no ambiente (GANDOLLA \& QUITIAN, 2005; GANDOLLA et al., 2009).

As diferentes formas de valorização e tratamento de resíduos conseguem reduzir a quantidade a ser encaminhada para um destino final mas não são capazes de eliminar a necessidade deste. É importante, então, planejar todo o sistema de gestão de resíduos de forma que o local de destino final gere menos impactos ao ambiente, natural e urbano, onde ele está inserido.

Deve-se ter como meta que o local de destinação final dos resíduos respeite, além de critérios de proteção ao ambiente natural ligados à geologia, hidrologia, geotecnia, meteorologia, entre outros, da área, também critérios relacionados ao ambiente urbano, como ocupação da área, degradação do entorno, tráfego de veículos, fluxos de entrada e saída de água, efluentes e energia e tempo para reutilização da área.

Quando esses critérios não são corretamente considerados, os locais de destino final de resíduos podem se tornar graves problemas para o ambiente e a sociedade. No Brasil, os muitos lixões ainda existentes pelo país refletem exemplos claros desses problemas. Mesmo na Europa, há exemplos de aterros mal planejados.

Vazamento de biogás a partir de aterros sanitários, colocando em risco habitações e indústrias vizinhas às áreas, foram identificados nos aterros de: Ghemme (Itália - 1992); Carate-Brianza (Itália - 1981); Casate (Suíça - 1981-1997); Augustin (Alemanha - 1981); Biella (Itália - 1981); Cavenago (Itália - 1996); Cerro (Itália - 1994-2000); Vergiate (Itália - 2001; culminando com a explosão de um prédio vizinho); Croglio (Suíça - 1992), como exemplos (ECONS, 2013).

Rupturas em aterros também aconteceram nos aterros de Sarajevo (Iugoslávia, 1977); culminando em um incêndio causado pelo vazamento de biogás), Istambul (Turquia, 1996) e Mariano Comense (Itália, 1992) (ECONS, 2013).

A fim de se evitar esses tipos de problemas, é importante que os novos aterros a serem construídos, tanto na Europa quanto no Brasil, sejam projetados como sendo realmente parte do planejamento urbano local.

\section{A incineração no sistema de gestão de resíduos}

O principal objetivo da incineração de resíduos é a redução do seu volume e da massa que será depositada em um aterro sanitário. A redução de 
massa pode variar entre 70 e $80 \%$, o que proporciona o aumento da vida útil dos aterros sanitários, que passam a receber apenas os rejeitos daqueles resíduos incinerados, conforme preconiza a Política Nacional de Resíduos Sólidos brasileira (HARRISON \& HESTER, 1994; CHIRICO, 1996; BRASIL, 2010).

O aumento da vida útil dos aterros sanitários é especialmente importante para as grandes metrópoles brasileiras, que não dispõem de área em seus territórios para construção de novos aterros e que encaminham seus resíduos para locais cada vez mais distantes dos centros geradores. O maior deslocamento dos resíduos até os locais de destinação final implica em elevação dos custos de operação do sistema de limpeza urbana associados ao transporte e à necessidade de construção de estações de transferência.

Os incineradores de resíduos também permitem recuperação energética, na forma de vapor e energia elétrica, a partir do calor liberado no processo de queima dos resíduos (TCHOBANOGLOUS et al., 1993; HARRISON \& HESTER, 1994; CHIRICO, 1996).

A recuperação energética de resíduos sólidos ganha importância quando analisado o cenário de demanda de energia elétrica no Brasil. De acordo com estudo da Empresa de Pesquisas Energéticas - EPE, o crescimento do consumo de energia elétrica crescerá $4,3 \%$ ao ano entre os anos de 2005 e 2030 (EPE, 2007). O consumo de energia elétrica na rede projetado para o Brasil para o ano de 2020 é de 659.092 GWh (EPE, 2011).

As unidades de incineração, quando bem planejadas em relação à sua integração urbana, podem gerar energia com distâncias reduzidas entre o local de geração e de consumo, reduzindo as perdas no transporte, com consequente ganho de eficiência no sistema.

Na Suíça, no ano de 2013, eram incineradas 3,79 milhões de toneladas de resíduos sólidos urbanos (não valorizáveis) em 30 plantas de incineração (ASED, 2014a). Essas plantas foram capazes de fornecer, em 2013, 1,96 GWh de vapor para calefação, 1,44 GWh de eletricidade e $703 \mathrm{GWh}$ de vapor para processos industriais (ASED, 2014a).

O Instituto Federal do Meio Ambiente da Suíça estima que, em 2012, a energia exportada pelas unidades de incineração era capaz de cobrir 2\% da demanda nacional (OFEV, 2014).

Embora eficientes na redução do volume e do peso dos resíduos e na produção de energia elétrica e térmica, os incineradores geram resíduos do processo que precisam ser adequadamente tratados e/ou dispostos. Esses resíduos são: gases gerados no processo de queima, que podem ser tóxicos; escória de material inerte não combustível; resíduos sólidos e líquidos dos processos de tratamento de gases; lixiviado, que pode ser gerado no local de acumulação de resíduos para queima (TCHOBANOGLOUS et al., 1993; HARRISON \& HESTER, 1994; CHIRICO, 1996).
Dessa forma, é relevante um bom planejamento na localização das unidades de incineração, além de um rigoroso controle operacional que garanta o correto funcionamento dos principais mecanismos de prevenção de poluição, a fim de se minimizar possíveis impactos ambientais negativos desse tipo de tratamento.

O Instituto Federal do Meio Ambiente da Suíça afirma, em um estudo publicado em 2008, que os incineradores naquele país emitem uma quantidade muito reduzida de poluentes atmosféricos, se comparados a outras fontes de poluição do ar. A emissão de partículas menores do que $10 \mathrm{~mm}$ (PM10) foi considerada desprezível em relação às demais emissões medidas no país. A emissão de $\mathrm{CO}_{2}$ na atmosfera seria de $1.271 \mathrm{~kg}$ por tonelada de resíduos incinerados, contribuindo com $3,5 \%$ do total de gases efeito estufa emitidos no país (OFEV, 2008).

$\mathrm{O}$ estudo ressalta ainda que as emissões de dioxinas, mercúrio e ácido clorídrico não podem ser desprezadas, embora não ultrapassem os limites estabelecidos pela legislação local de controle de poluição do ar (OFEV, 2008).

Dados disponibilizados pela empresa Usina de Valorização Térmica e Elétrica de Resíduos TRIDEL (Lausanne, Suíça) mostram que nos anos de 2011, 2012 e 2013, os parâmetros ácido clorídrico, ácido fluorídrico, dióxido de enxofre, nitrogênio amoniacal, mercúrio, cádmio, chumbo+zinco e monóxido de carbono, medidos nas emissões gasosas das duas linhas existentes na planta, estiveram no mínimo $80 \%$ abaixo dos valores limites estabelecidos pela legislação de controle de poluição do ar Suíça e também abaixo dos limites definidos pela legislação europeia (TRIDEL, 2014). A quantidade de dióxido de nitrogênio esteve, no mesmo período, ao menos $40 \%$ reduzida em relação à legislação local (TRIDEL, 2014).

Em relação à escória gerada pelos materiais inertes não combustíveis, ela segue para recuperação de metais ferrosos e não ferrosos, devido à demanda existente e ao elevado valor de mercado desses materiais (OFEV, 2008). A parcela não recuperável segue para aterros de materiais inertes.

Acerca dos custos das unidades de incineração, é difícil definir valores que possam ser utilizados de forma universal, pois há significativas variações entre custos do terreno, requisitos estruturais devido ao clima (temperaturas externas muito baixas, neve, etc.), custos de materiais e equipamentos, valor de mão de obra, etc.

Apenas como referência, no ano de 2008, na Suíça, a operação dessas unidades custou em média SFr 40 (R\$121,60) por habitante. O custo de transporte dos resíduos até as unidades de incineração custou SFr $21(\mathrm{R} \$ 63,84)$ a cada habitante e a coleta seletiva mais a valorização dos materiais recicláveis e orgânicos, SFr 45 ( R\$ 136,80) por habitante (OFEV, 2009).

Os valores cobrados para recebimento de resíduos nas unidades de incineração na Suíça, em fevereiro de 2014, variavam entre SFr 100 (R\$ $304,00)$ e SFr 200 (R\$608,00) por tonelada (ASED, 2014b). 
Embora os valores exatos de custos de incineração não possam ser definidos, é possível fazer uma comparação com outros tipos de tratamento e destino final de resíduos.

Proporcionalmente, a incineração possui custo de implantação mais alto do que aterros sanitários, que podem ser considerados de baixo custo de implantação. O custo de gestão dos incineradores também pode ser considerado alto em relação aos aterros, que possuem custos menores de gestão. Para o custo de encerramento, as unidades de incineração possuem custo baixo quando comparadas aos aterros, que possuem custo de encerramento alto. O custo de monitoramento e manutenção pós-encerramento dos incineradores é praticamente nulo, enquanto que o dos aterros, muito alto.

$\mathrm{Na}$ Suíça, as plantas de incineração são administradas por empresas de capital público, o que permite amplo acesso a informações pela população e observância aos interesses comuns. Tais empresas possuem estrutura administrativa semelhante à de empresas privadas, sendo constituídas como sociedades anônimas (SA), porém são oriundas de consórcios entre municípios e estados.

O fato de empresas que operam as plantas de incineração serem sociedades anônimas reduz a ingerência política sobre as mesmas, de forma que a sua continuidade operacional independe das mudanças de governo. No caso da implantação de incineradores no Brasil, essa continuidade operacional precisa ser fortemente observada de forma que se possa garantir um rigoroso controle ambiental e de produção energética nas unidades.

\section{CONCLUSÕES}

Assim como a Europa há algumas décadas, o Brasil está começando um processo de mudança na gestão dos seus resíduos sólidos urbanos. O encerramento gradual das áreas de disposição inadequada de resíduos e a busca por novas soluções para otimizar e tornar mais sustentável a sua gestão começam a ser percebidos em algumas cidades do país.
No Brasil, a geração de resíduos é crescente, o que demanda cada vez mais recursos financeiros e pessoal para todas as etapas do processo de gestão. É importante observar as medidas que foram adotadas na Europa, a fim de responsabilizar a população e os fabricantes de materiais pelos custos de recolhimento, valorização e destinação dos materiais pós-consumo, que devem ter contribuído para a redução da geração de resíduos, e avaliar como medidas semelhantes podem ser adotadas no Brasil.

A sustentação financeira é fundamental para que possam ser implantados e mantidos os padrões necessários de qualidade operacional nos municípios, com a contribuição e participação efetiva da população geradora dos resíduos (JUCÁ et al., 2014).

Do ponto de vista da gestão dos resíduos sólidos, é preciso integrar as suas diferentes etapas, desde a acumulação até a destinação final, ao planejamento urbano a fim de reduzir os impactos da operação, encerramento e pós-encerramento dessas atividades, bem como permitir a reurbanização das áreas usadas para os tratamentos e destinação dos residuos.

Na Europa, existem dificuldades maiores para essa integração, ligadas à concentração populacional, ao território reduzido, ao clima variável, etc.. No Brasil, onde as unidades de tratamento e destinação de resíduos começam a ser planejadas e onde as dificuldades citadas não são tão presentes, é importante que elas sejam projetadas em consonância com o planejamento urbano das cidades. Ao se avaliar as experiências positivas e negativas que ocorreram em outros locais, pode-se evitar repetir erros já ocorridos.

A incineração, como tratamento de resíduos sólidos, pode vir a fazer parte da gestão de resíduos no Brasil, assim como faz na Europa. Ela permite otimizar o uso dos aterros sanitários e recuperar energia, fatores importantes também para o Brasil. Além disso, permite reduzir os custos de gerenciamento e os impactos ambientais dos resíduos para as próximas gerações. Entretanto, além de serem projetadas de acordo com o planejamento urbano, essas unidades precisam manter um rigoroso controle operacional e de prevenção de poluição de forma a contribuirem positivamente para o sistema de gestão de resíduos.

\section{REFERÊNCIAS}

ABRELPE - ASSOCIAÇÃO BRASILEIRA DE EMPRESAS DE LIMPEZA PÚBLICA E RESÍDUOS ESPECIAIS. Panorama dos Resíduos Sólidos no Brasil 2003. São Paulo: ABRELPE, 2004.

ABRELPE - ASSOCIAÇÃO BRASILEIRA DE EMPRESAS DE LIMPEZA PÚBLICA E RESIDUUS ESPECIAIS. (2014) Panorama dos Resíduos Sólidos no Brasil 2013. São Paulo: ABRELPE.

ASED - ASSOCIATION SUISSE DES EXPOITANTS D'INSTALLATIONS DE TRAITEMENT DES DECHETS. (2014a) Energie-Charts. Disponível em: <http:// vbsa.ch/fr/donnees/energie-charts/> Acesso em: 02 jul 2014.
ASED - ASSOCIATION SUISSE DES EXPOITANTS D'INSTALLATIONS DE TRAITEMENT DES DECHETS. (2014b ) Tarifs d'incinération 20122014 pour les déchets urbains des communes actionnaires, par installation. Disponível em: <http://vbsa.ch/fr/tarifs-dincinerationde-dechets-urbains-des-communes-actionnaires-par-installation/>. Acesso em: 02 jul 2014.

BRASIL. (2010) Lei Federal no 12.305 - Institui a Política Nacional de Resíduos Sólidos; altera a Lei no 9.605, de 12 de fevereiro de 1998; e dá outras providências. Diário Oficial [da União], O3 de agosto de 2010. 
CHIRICO, V. (1996) Municipal Waste Treatment Plants. Zurich: Swiss Reinsurance Company.

ECONS - ENVIRONMENTAL ENGINEERING. (2013) Safety in landfills: photographic documentation. Disponivel em: <http://www.econs.ch/econs. php?mp=pubblicazioni> Acesso em: O2 jul 2014.

EPE - EMPRESA DE PESQUiSAS ENERGÉTICAS. (2007) Plano Nacional de Energia 2030. Apresentação Mauricio Tolmasquim, Presidente EPE. Brasília: EPE.

EPE - EMPRESA DE PESQUISAS ENERGÉTICAS. (2011) Projeção da demanda de energia elétrica para os próximos 10 anos (2011-2020). Nota Técnica DEA 03/11. Brasília: EPE, 2011.

EUROSTAT - ENVIRONMENTAL DATA CENTRE ON WASTE. (2O11) Statistics in Focus no 31/2O11. Disponível em: <http://epp.eurostat.ec.europa.eu/cache/ ITY_OFFPUB/KS-SF-11-O31/EN/KS-SF-11-O31-EN.PDF>. Acesso em: 14 ago 2013

EUROSTAT - ENVIRONMENTAL DATA CENTRE ON WASTE. (2014a) Disponível em: <http://epp.eurostat.ec.europa.eu/portal/page/portal/waste/ data/database>. Acesso em: O2 jul 2014.

EUROSTAT - ENVIRONMENTAL DATA CENTRE ON WASTE. (2014b) Eurostat News Release no 48/2014. Disponível em: <http://epp.eurostat. ec.europa.eu/portal/page/portal/publications/collections/news_releases> Acesso em: O2 jul 2014.

GANDOLLA, M \& QUITIAN, M.G. (2005) Criterios de planificación de un vertedero de residuos moderno. II ATEGRUS/ISWA Beacon Conference on Hazardous Wastes.

GANDOLLA M.; QUITIAN, M.G.; ROBLEDO, E. (2009) Criterios de planificación de un moderno vertedero basado sobre el análisis del metabolismo urbano. 90 Congreso Internacional Disposición Final de Residuos y Perspectivas Ambientales.

JUCA, J.F.T;; LIMA, J.D.; LIMA, D.A.; MARIANO M.O.; LUCENA L.; FIRMO, A. (2014) Análise das diversas tecnologias de tratamento e disposição final de resíduos sólidos urbanos no Brasil, Europa, Estados Unidos e Japão. Jaboatão dos Guararapes (PE): UFPE - BNDES, CCS Gráfica Editora Ltda.

HARRISON, R.M. \&; HESTER, R.E. (1994) Waste Incineration and the Environment. Royal Society of Chemistry.

IBGE - INSTITUTO BRASILEIRO DE GEOGRAFIA E ESTATISTICA. (2010) Pesquisa Nacional de Saneamento Básico 2008. Rio de Janeiro: IBGE.
OFEV - OFFICE FEDERAL DE L'ENVIRONNEMENT. (2008) Rapport sur la gestion des déchets 2008. Politique suisse de gestion des déchets 2005-2007: données et perspectives. Etat de l'environnement no 0830. OFEV.

OFEV - OFFICE FEDERAL DE L'ENVIRONNEMENT. (2009) Magazine «environnement» 3/2009 - Déchets: une mine d'or. OFEV.

OFEV - OFFICE FEDERAL DE L'ENVIRONNEMENT. (2O11) Combustible waste and MSWI capacity 1996 2O1O. OFEV.

OFEV - OFFICE FEDERAL DE L'ENVIRONNEMENT. (2O13) Magazine «environnement» 3/2013 - La ronde des ressources. OFEV.

OFEV - OFFICE FEDERAL DE L'ENVIRONNEMENT. (2014) Rapport d'état Gestion des déchets. OFEV.

SUISSE - CONSEIL FEDERAL SUISSE. (1990) Loi 814.600 Ordonnance sur le traitement des déchets, 10 décembre 1990.

TCHOBANOGLOUS, G.; THEISEN, H.; VIGIL, S.A. (1993) Integrated Solid Waste Management - Engineering Principles and Management Issues. McGraw-Hill International Editions.

TRIDEL - USINE DE VALORISATION THERMIQUE ET ÉLECTRIQUE DE DECHETS. (2014) Emissions à la cheminée - tableau comparatif 2011/2012/2013. Disponível em: <http://www.tridel.ch/ environnement/protection/air.html> Acesso em : 02 jul 2014

UE - UNIÃO EUROPÉIA. (1994) Conselho da União Europeia. Directiva 94/62/EC - Relativa à embalagens e resíduos de embalagens, 20 de dezembro de 1994.

UE - UNIÃO EUROPÉIA UE. (1999) Conselho da União Europeia. Directiva 1999/31/EC - Relativa à deposição de resíduos em aterros, 26 de abril de 1999.

UE - UNIÃO EUROPÉIA UE. (2000) Conselho da União Europeia. Directiva 2000/76/EC - Relativa à incineração de resíduo. 04 de dezembro de 2000

UE - UNIÃO EUROPÉIA UE. (2006) Conselho da União Europeia. Directiva 2006/12/EC - Relativa aos resíduos. 05 de abril de 2006.

UE - UNIÃO EUROPÉIA. (2008) Conselho da União Europeia. Directiva 2008/98/EC - Relativa aos resíduos e que revoga certas directivas. 18 de novembro de 2008. 